\title{
A Robotic Wide-Angle H $\alpha$ Survey of the Southern Sky
}

John E. Gaustad

Dept. of Phys. \& Astr., Swarthmore Coll., Swarthmore PA 19081, USA

Wayne Rosing

Las Cumbres Obs., 1500 Miramar Beach, Montecito CA 93108, USA

Peter McCullough

Dept. of Astronomy, Univ. of Illinois, Urbana IL 51801, USA

Dave Van Buren

Extrasolar Research Corporation, Niskayuna NY 12309, USA

\begin{abstract}
We are conducting a robotic wide-angle imaging survey of the southern sky at $656.3 \mathrm{~nm}$ wavelength, the $\mathrm{H} \alpha$ emission line of hydrogen. Each image of the survey covers an area of the sky $13^{\circ}$ square at an angular resolution of approximately one arcminute, and reaches a sensitivity level of 0.5 Rayleigh $\left(3 \times 10^{-18} \mathrm{erg} \mathrm{cm}^{-2} \mathrm{~s}^{-1} \operatorname{arcsec}^{-2}\right)$, corresponding to an emission measure of $1 \mathrm{~cm}^{-6} \mathrm{pc}$, and to a brightness temperature for microwave free-free emission of $3 \mu \mathrm{K}$ at $30 \mathrm{GHz}$.
\end{abstract}

\section{Scientific Purpose}

This survey will produce detailed information on the structure of the diffuse, warm, ionized component of the interstellar medium, information necessary for understanding the dynamics and evolutionary history of the interstellar gas.

The intensity of the microwave free-free emission from Galactic hydrogen is directly proportional to the brightness at $\mathrm{H} \alpha$. Thus these images will also allow measurement of anisotropies in the Galactic free-free emission at microwave wavelengths (or proof that these are negligible), emission which must be subtracted to obtain the true cosmic background anisotropies.

\section{Instrumentation and Observations}

Our CCD camera, located at Cerro Tololo Inter-American Observatory, contains a $1024 \times 1024$ TI chip with $12-\mu \mathrm{m}$ pixels. The sky is imaged onto the chip with a $52-\mathrm{mm}$ f.l. lens operated at $\mathrm{f} / 1.6$, yielding a field of view of $13^{\circ} \times 13^{\circ}$ and a scale of $48^{\prime \prime} /$ pixel. A filter wheel mounted in front of the lens contains an $\mathrm{H} \alpha$ filter of 3-nm bandwidth and a dual-band notch filter, which excludes $\mathrm{H} \alpha$ but transmits two 6-nm bands of continuum radiation on either side of $\mathrm{H} \alpha$. The 


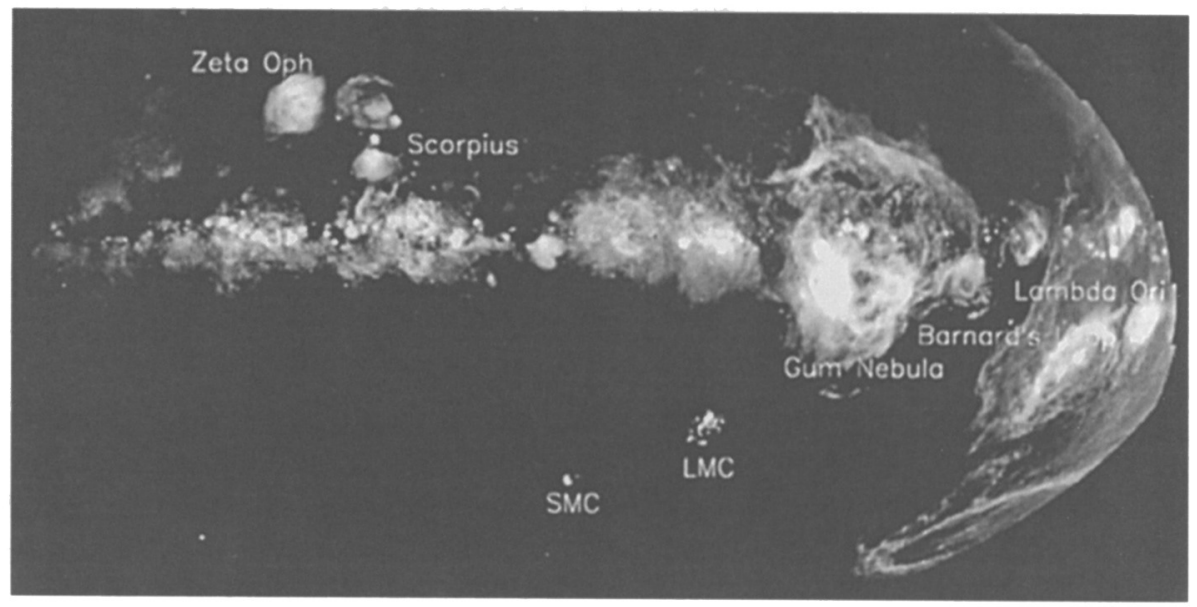

Figure 1. A mosaic of the southern sky in $\mathrm{H} \alpha$. See also front cover of the volume.

unguided tracking accuracy of our Byers Series 2 German mount is better than 2 arcseconds per hour. Thus no external guider is needed.

We can inquire of the status of the robot via email, and make changes in the program or observing schedule via ftp. But we do not operate the camera in real time - it is a true robot. The robot has its own weather station, but as an extra precaution it asks permission (via email) of the 4-meter telescope operator before opening the dome.

All observations are taken after twilight and with the moon below the horizon. A normal set of science observations consists of five 20-minute exposures through the $\mathrm{H} \alpha$ filter interspersed among six 5-minute exposures through the dual-band continuum filter.

\section{Survey Status}

The survey consists of 283 fields covering the sky from $-90^{\circ}$ to $+15^{\circ}$ declination, with the same centers as those in the IRAS Sky Survey Atlas. In order to reduce the noise and to confirm objects discovered at the faintest brightness levels, we repeat all fields with $5^{\circ}$ offsets. The data-gathering phase of the project was completed in October 2000, and the survey is expected to be published by summer 2001. A mosaic, in galactic coordinates, is shown in Figure 1.

\section{Acknowledgements}

The project has been supported by grants from Las Cumbres Observatory, the National Science Foundation, NASA, Dudley Observatory, the Fund for Astrophysical Research, Research Corporation, the University of Illinois, and Swarthmore College. 